\title{
Associative Memories with Small World Connectivity
}

\author{
Neil Davey, Lee Calcraft, Bruce Christianson, Rod Adams \\ Department of Computer Science, University of Hertfordshire, UK \\ E-mail: \{N.Davey, L.Calcraft, B.Christianson, R.G.Adams\}@herts.ac.uk
}

\begin{abstract}
In this paper we report experiments designed to find the relationship between the different parameters of sparsely connected networks of perceptrons with small world connectivity patterns, acting as associative memories.
\end{abstract}

\section{Introduction}

It is possible to build associative memory models from networks of simple perceptrons. These networks perform much better than the canonical Hopfield model, both in terms of capacity and pattern completion. It is also possible to use such networks of perceptrons with sparse or diluted connectivity, and the performance is relatively robust, even at high rates of dilution. Of course real neural networks have sparse connectivity (for example in the cortex of the mouse each neuron is connected to about $0.1 \%$ of the other neurons [1]), which motivates the investigation undertaken here. It is also known that in biological systems the networks have a small world characteristic $[2,3]$. That is they exhibit short path lengths (the minimum number of nodes on a path) between any pair of neurons, as in a random network, but also show a cliquish behaviour, with locally clustered connections. The advantage of such clustering is apparent in the mean connection length (the average wiring length), which is far smaller than in an equivalent random network, see Figure 1. A further benefit is that the wiring complexity is much reduced in networks with predominantly local connections. Recent research has shown that a small world Hopfield network could be a relatively effective associative memory [4]. In our earlier paper [5] we showed how the high capacity model could benefit from small world connectivity. Here we investigate the detailed relationship between the nature of the connection graph, the size of the network and the resulting performance. Sections 2 and 3 describe the background and the computational model. After the performance measure is explained in Section 4 the results are given in Section 5. The paper finishes with a discussion.

\section{Background}

The simple small world model of Watts and Strogatz [2] consists of a regular $N$-node ring lattice. Each node is connected to $k / 2$ neighbours on either side, where $k$ is typically small compared to $N$. The mean path length between any pair of random points is therefore high. A fraction, $p$, of these local connections is then rewired to randomly selected nodes, see right diagram in Figure 1. They showed that at surprisingly low values of $p$, the mean path length in the network dropped dramatically, resulting in a small world regime: highly clustered but with low path lengths. Many real networks have been shown to have a small world architecture, including the internet, human acquaintance networks and real networks of neurons [2]. Theoretical work [6] has now shown the detailed relationship between the characteristics of such networks.

Much is known about the effect of sparse connectivity on the standard Hopfield neural network. Some work has also been undertaken on diluted Hopfield networks with modular and small world connectivity [4, 7-9].

For the higher capacity version of the Hopfield network, trained using perceptron learning much less is known about the effect of connectivity patterns. It was shown in [10] that capacity falls linearly with dilution and in [11] that structured local connectivity could help in storing locally correlated data.

The sparse network with only local connectivity can be considered as a simple example of a Cellular Neural Network and it has been proposed [12] that such networks can be used as associative memories.
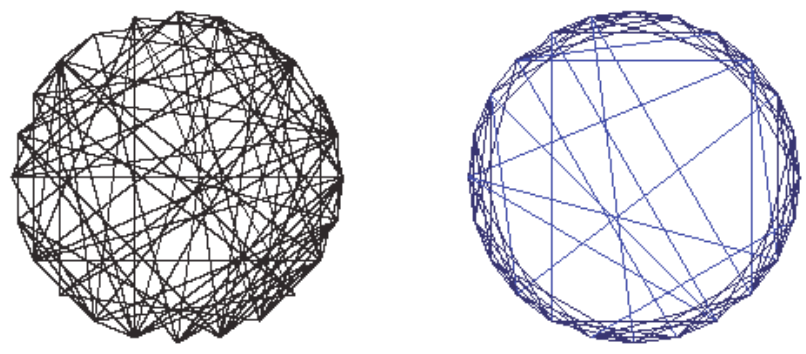

Fig. 1. A ring, with random connectivity on the left and small world connectivity on the right. 


\section{Network Model}

The high capacity models studied here are a modification of the standard Hopfield network. The net input, or local field, of a unit, is given by: $h_{i}=\sum_{j \neq i} w_{i j} S_{j}$ where $\mathrm{S}( \pm 1)$ is the current state and $w_{i j}$ is the weight on the connection from unit $j$ to unit $i$. The dynamics of the network is given by the standard update: $S_{i}^{\prime}=\Theta\left(h_{i}\right)$, where $\Theta$ is the heaviside function. Unit states may be updated synchronously or asynchronously. Here we use asynchronous, random order updates. If a training pattern $\xi^{\mu}$ is a fixed point of the dynamics then it is successfully stored, and is said to be a fundamental memory. A network state is stable if, and only if, all the local fields are of the same sign as their corresponding unit, equivalently the aligned local fields, $h_{i} S_{i}$, should be positive.

We examine sparse networks with small world connectivity. The network topology is similar to the original Watts and Strogatz model. We start with an $\mathrm{N}$ ring regular lattice, with each unit connected to its $k$ nearest neighbours, and then rewire with probability $p$. However as a network of perceptrons is not necessarily constrained to have symmetric connections we can generalize the network to a weighted directed graph (as is the case for real neural networks). The rewiring does not therefore maintain the symmetry of connectivity of the original regular lattice. In fact it has been shown [5] that for sparse networks of this type, symmetric weights give rise to poor performance.

The networks are trained using the normal perceptron training rule:

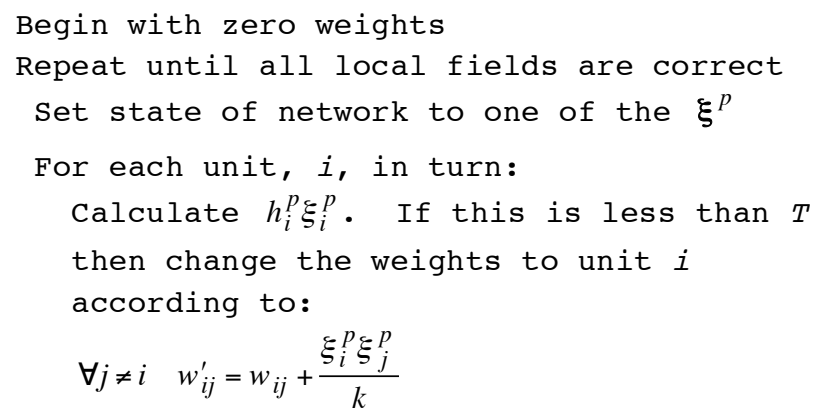

Where $\xi^{p}$ denotes the training patterns, and $T$ is the learning threshold which here has the value of 10 .

\section{Performance Measure}

We are interested in how well the small world networks and random networks, trained using the perceptron style learning rule described above, perform as associative memories. The capacity of such networks is determined by the number of incoming connections $(k)$ that each perceptron has. For random pattern sets a perceptron can learn up to $2 k$ patterns [13]. Assuming roughly regular connectivity graphs (as is the case here) the capacity will be determined by the level of dilution and not the specific pattern of connections, and hence is not subject to empirical investigation.

We use $R$, the normalised mean radius of the basins of attraction, as a measure of attractor performance in these networks. It is defined as:

$$
R=\left\langle\left\langle\frac{1-m_{0}}{1-m_{1}}\right\rangle\right\rangle
$$

where $m_{0}$ is the minimum overlap an initial state must have with a fundamental memory for the network to converge on that fundamental memory, and $m_{1}$ is the largest overlap of the initial state with the rest of the fundamental memories. The angled braces denote a double average over sets of training patterns and initial states. Details of the algorithm used can be found in [10]. A value of $R=1$ implies perfect performance and a value of $R=0$ implies no pattern correction.

\section{Results}

In this paper we report experiments designed to find the relationship between the different parameters of the model. We summarise these parameters in Table 1.

Table 1: Parameters of the Model

\begin{tabular}{|l|l|}
\hline$N$ & The size of the network \\
\hline$k$ & The number of connections each unit makes \\
\hline$\alpha$ & The loading per connection: size of training set / $k$ \\
\hline$p$ & The proportion of rewired connections \\
\hline
\end{tabular}

\subsection{Fixed Size Networks}

The first set of experiments fixes the network size, $N$, at 1000 units, arranged in a ring as described earlier. Initially each unit is connected to its $k=20,40$ or 60 neighbours. Random training sets of 1000-ary vectors are created. The number of vectors in the training set is determined by the specific values of $\alpha$ and $k$. For example with $\alpha=0.3, k=20$ implies a training set of 6 vectors, $k=4012$ vectors and $\mathrm{k}=6018$ vectors (as used in Figure 3$)$. The attractor performance ( $R$ value) is then measured as the network is progressively rewired, as described above. All results presented are averages over 10 runs.

Figure 2 gives the results. Considering first the overall pattern, it can be seen that, in all cases, as the amount of rewiring is increased the performance of the network is also improved. In fact all the networks reach a point at 
which pattern correction behavior is perfect. It is also apparent that higher levels of rewiring are required as the loading, $\alpha$, of the networks is raised.
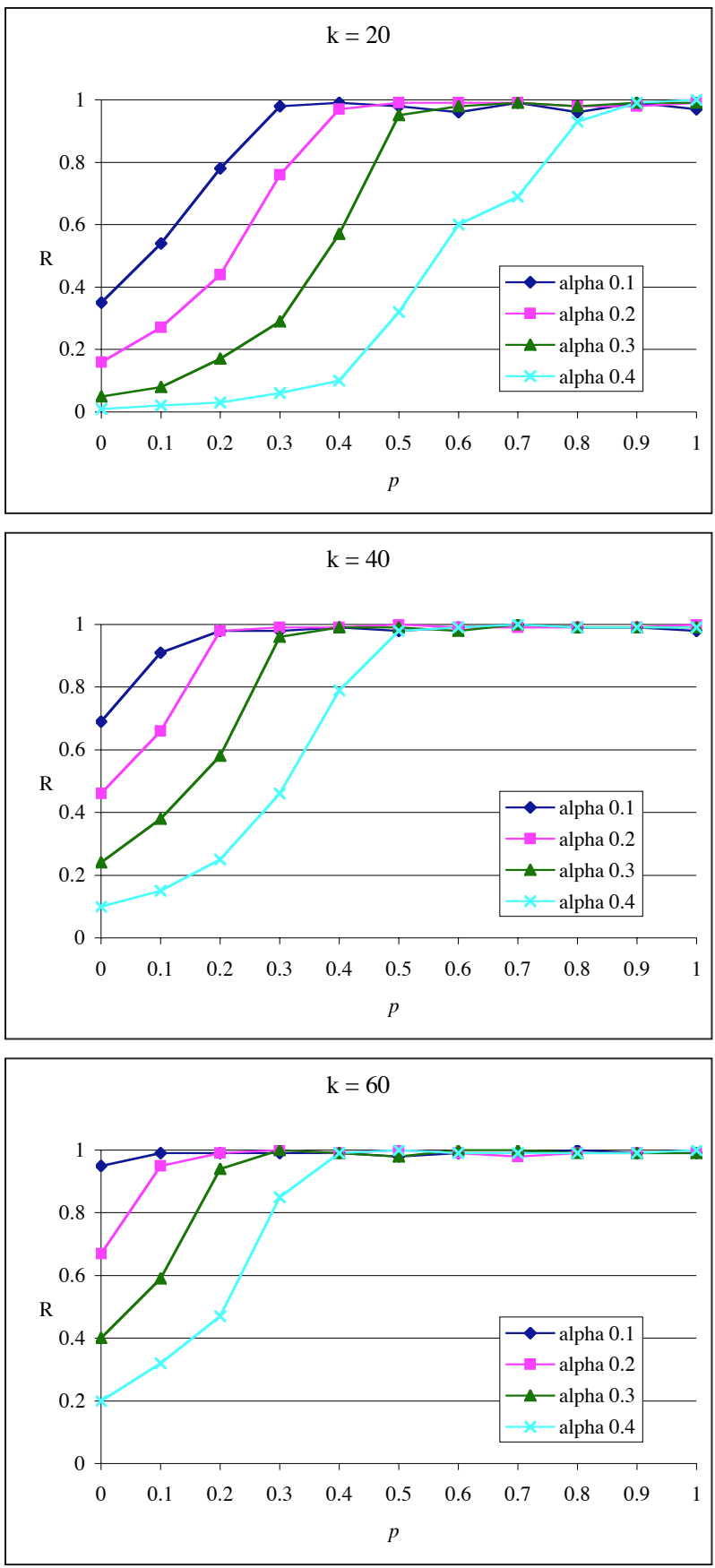

Fig. 2. Networks of size $N=1000$. The attractor performance $R$ is reported for different values of connectivity $k$, and at varying loadings, $\alpha$, as the networks are progressively rewired, by amount $p$. Results are averages over 10 runs.

Now examining the comparative performance at different levels of connectivity it can be seen that, for a given loading, the larger the value of $k$ the better the network performs. Figure 3 gives a different view of this, fixing the loading at 0.3 , and plotting $R$ for different $k$ values on the same graph.

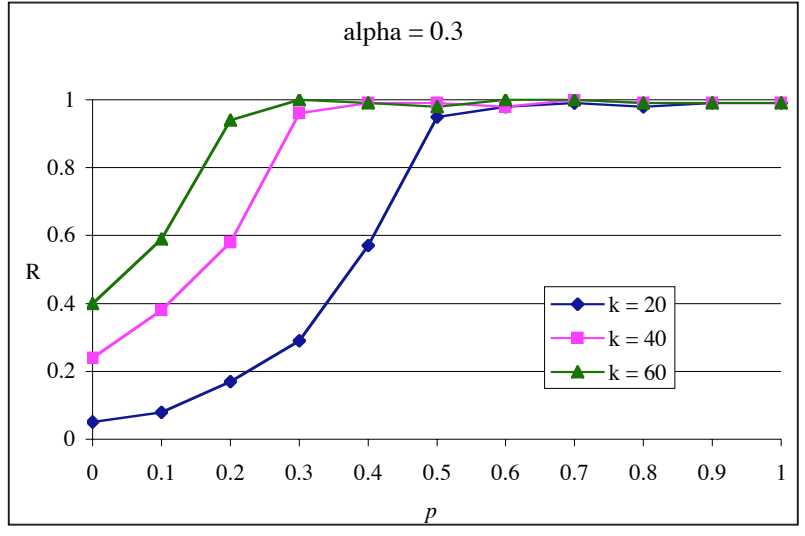

Fig. 3. A different view of the results in Figure 2. $\alpha$ is fixed at 0.3 . It can be seen how the higher values of $k$ give better performance.

\subsection{Size Effects}

The next set of results examines how increasing the size of the network by an order of magnitude affects the performance of the network. Figure 4 summarises the results, here the loading on the networks is fixed at $\alpha=$ 0.3. The lower two plots on the graph show the performance of networks of sizes $N=1000$ and $N=$ 10,000 with purely local connectivity $(p=0)$. As observed above increasing the level of (local) connectivity improves performance. The larger network has a poorer attractor performance than the $N=1000$ version, although when $k$ reaches 800 a point of good performance is reached. The explanation for both these observations is probably that if the size of the network is relatively large, when compared to the local neighbourhood of each unit, then it is progressively harder for information to propagate through the network. In other words the more local the connectivity relative to the size of the network the more difficult is it to make a successful global computation such as pattern correction.

The upper pair of lines shows the dramatic effect of just $10 \%$ rewiring. Once again the smaller network initially has better performance, but by $k=180$ the $N=10,000$ network is performing almost perfectly. In this configuration the network is storing 54 10,000-ary vectors, with each unit having only 180, mostly local, connections and is still able to perform almost perfect pattern completion. This architecture is extremely parsimonious. The mean wire length is only about $12 \%$ of that of a random network with the same level of connectivity. 


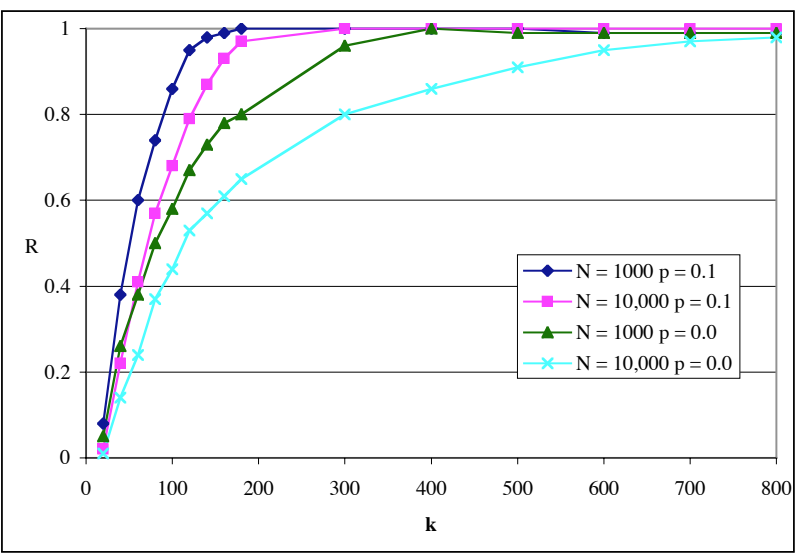

Fig. 4. Networks of size $N=1000$ and $N=10,000$, either with local connectivity only $p=0$ (lower two plots), or with $p=0.1$, (upper two plots). The attractor performance $R$ is reported for different values of connectivity $k$. In all cases the loading of the network is $\alpha=0.3$. Results are averages over 10 runs.

\section{Discussion}

The fully connected models of associative memory, such as the standard Hopfield network are difficult to realize in VLSI implementations and are not viable as abstract models of real neural networks, which have sparse connectivity. Locally connected associative memories have been proposed as possible solutions to the first of these issues [12]. However as our results show the pattern correction ability of networks with only local connections is compromised. In order to restore the performance of locally connected networks to that of an equivalent random network it is necessary to introduce some rewiring - to change the pattern of connectivity to one of a small world graph. The results show that the amount of rewiring required is dependant upon both the relative level of connectivity and the loading on the network. More rewiring is required with either increased loading or decreased relative connectivity. However the very large networks of 10,000 units performed very well with only $10 \%$ rewiring at a loading of 0.3 . In fact looking at relative connectivity the 10,000 unit network can be thought of as performing better than the 1000 unit network, since it produces perfect pattern completion with a much lower ratio of connections to network size.

Perhaps the most interesting feature of these small world networks is that they suggest how global computation is accomplished in real networks of neurons where distal connectivity comes at considerable cost.

Further work will explore other types of small world connectivity patterns, such as scale free networks [8]. We are also interested in finding the pattern of connectivity that maximizes performance whilst minimizing wiring cost.

\section{References}

1. Braitenberg, V. and A. Schüz, Cortex: Statistics and Geometry of Neuronal Connectivity. 1998, Berlin: Springer-Verlag.

2. Watts, D. and S. Strogatz, Collective Dynamics of 'small-world' networks. Nature, 1998. 393: p. 440-442.

3. Shefi, O., et al., Morphological characterization of in vitro neuronal networks. Physical Review E, 2002. 66(021905).

4. Bohland, J. and A. Minai Efficient Associative Memory Using Small-World Architecture. Neurocomputing, 2001. 38-40: p. 489-496.

5. Davey, N., B. Christianson, and R. Adams. High Capacity Associative Memories and Small World Networks. in IJCNN. 2004. Budapest.

6. Newman, M.E.J., Models of the Small World. Journal of Statistical Physics, 2000. 101(3/4): p. 819-841.

7. McGraw, P. and M. Menzinger, Topology and computational performance of attractor neural networks. Physical Review E, 2003. 68: p. 047102.

8. Stauffer, D., et al., Efficient Hopfield pattern recognition on a scale-free neural network. European Physical Journal B, 2003. 32(3): p. 395-399.

9. Torres, J.J., et al., Influence of topology on the performance of a neural network.

Neurocomputing, 2004. 58-60: p. 229-234.

10. Davey, N. and R. Adams, High Capacity Associative Memories and Connection Constraints. Connection Science, 2004. 16(1): p. 47-66.

11. Turvey, S.P., PhD Thesis: Analysing and Enhancing the Performance of Associative Memory Architectures, in Computer Science. 2003, University of Hertfordshire: Hatfield.

12. Brucoli, M., L. Carnimeo, and G. Grassi. Discrete-time cellular neural networks for associative memories: a new design method via iterative learning and forgetting algorithms. in 38th Midwest Symposium on Circuits and Systems. 1995.

13. Hertz, J., A. Krogh, and R.G. Palmer, Introduction to the Theory of Neural Computation. 1991, Redwood City, CA: Addison-Wesley Publishing Company. 327. 